\title{
Fundamental limits on detection in low SNR under noise uncertainty
}

\author{
Rahul Tandra \\ Department of Electrical and Computer Science \\ University of California, Berkeley \\ Berkeley, CA 94720 \\ Email: tandra@eecs.berkeley.edu
}

\author{
Anant Sahai \\ Department of Electrical and Computer Science \\ University of California, Berkeley \\ Berkeley, CA 94720 \\ Email: sahai@eecs.berkeley.edu
}

\begin{abstract}
In this paper we consider the problem of detecting whether a frequency band is being used by a known primary user. We derive fundamental bounds on detection performance in low SNR in the presence of noise uncertainty - the noise is assumed to be white, but we know its distribution only to within a particular set. For clarity of analysis, we focus on primary transmissions that are BPSK-modulated random data without any pilot tones or training sequences. The results should all generalize to more general primary transmissions as long as no deterministic component is present.

Specifically, we show that for every 'moment detector' there exists an SNR below which detection becomes impossible in the presence of noise uncertainty. In the neighborhood of that SNR wall, we show how the sample complexity of detection approaches infinity. We also show that if our radio has a finite dynamic range (upper and lower limits to the voltages we can quantize), then at low enough SNR, any detector can be rendered useless even under moderate noise uncertainty.
\end{abstract}

\section{INTRODUCTION}

Detection has a wide range of applications including communications. [11] is one of the early efforts highlighting the importance of this field and [8] provides a good survey of the results and techniques of detection theory developed in the past decades. In many cases, the receiver knows the signal and hence the optimal detector is just a matched filter [9]. In this case it is well known that the number of samples required to hit a target probability of false alarm and missed detection grows as $O(1 / S N R)$ when the SNR is low. If the receiver only knows the power of the signal then the optimal detector is just an energy detector (radiometer) [13], [16]. In this case, $O\left(1 / S N R^{2}\right)$ samples are required to meet a particular performance target.

Our interest in the very low SNR regime is motivated by the possibility for cognitive radios [4], [10]. This represents a new paradigm for spectrum utilization in which new devices can opportunistically scavenge bands that are not being used at their current time and location for their primary purpose [3]. This is inspired by actual measurements showing that most of the allocated spectrum is vastly underutilized [2].

However, fundamental theoretical questions remain as to the exact requirements for engineering a practical cognitive radio system so that they do not interfere with the primary users. An introduction to the tradeoffs and challenges faced by cognitive radios can be found in [12]. In particular, in order to guarantee non-interference with primary users ${ }^{1}$ without being restricted to very low transmit powers, the cognitive radio system needs to be able to detect the presence of very weak primary signals. [7]

In this paper, our interest is in what happens in the low SNR regime when we consider the Gaussian noise assumption as holding only approximately. [14] analyzes the affect of noise power uncertainty in radiometric detection of spread-spectrum signals and identifies a fundamental limit on the SNR of the signals we can detect. We extend their results beyond the energy detector to detectors that examine other moments. More importantly, we show that the fundamental limits on moment detectors become hard limits on any possible detector if the radio has a finite dynamic range on its input. Since all physical radios have such a limit, we feel that the bounds here represent important constraints on practical systems.

\section{BACKGROUND REVIEW}

We consider the problem of detection of a weak BPSK signal $(X)$ in additive white Gaussian noise (AWGN) $(W)$. Our goal is to distinguish between the hypotheses:

$$
\begin{array}{ll}
\mathcal{H}_{0}: Y[n]=W[n] & n=1, \ldots, N \\
\mathcal{H}_{1}: Y[n]=X[n]+W[n] & n=1, \ldots, N
\end{array}
$$

Since we are interested in the low SNR regime, the number of samples required is large. Thus, we can use the central limit theorem (see [1]) to approximate the log-likelihood test statistic as a Gaussian. Fig. 1 shows the performance curve of the optimal detector. Note that the optimal detector performs as badly as the energy detector. However, many communication schemes have training sequences which can act as weak pilot signals if their structure is perfectly known. By designing a suboptimal detector that just searches for these pilots, we can substantially reduce the number of samples required to detect when the SNR is low (see Fig. 1). Furthermore, these observations hold for general zero-mean signal constellations also, as long as the signal power $P$ is weak [12].

\footnotetext{
${ }^{1}$ This is a version of the hidden terminal problem in which the primary system might have a receiver vulnerable to secondary interference while simultaneously the primary transmissions are shadowed enroute to the secondary user.
} 


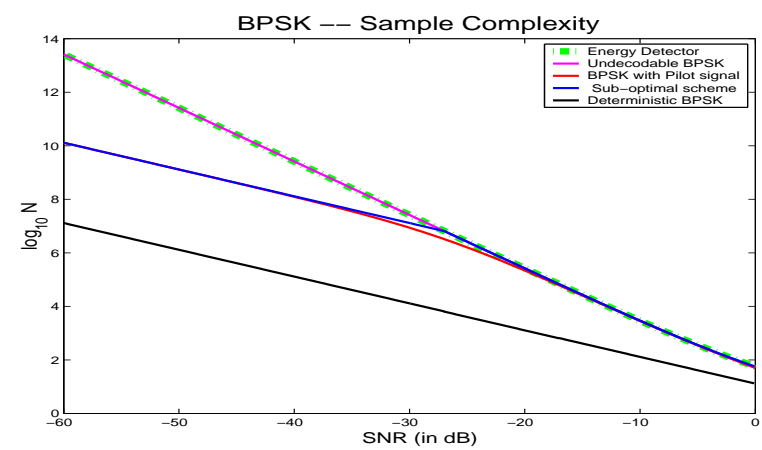

Fig. 1. The figure compares the sample complexity curves for an undecodable BPSK signal without a pilot and the sample complexity curves of an undecodable BPSK signal with a known pilot signal. The dashed green curve shows the performance of the energy detector, the pink curve corresponds to the performance of the optimal detector. Both these curves are for the case without a pilot signal. These curves show that the energy detector performance is same as that of the optimal detector. The red curve gives the performance of the optimal detector in the presence of a known weak pilot signal. Note, that there is a significant decrease in sample complexity due to the known pilot signal, especially at very low SNR's. Specifically, at low SNR, the sample complexity changes from $O\left(1 / S N R^{2}\right)$ to $O(1 / S N R)$ due the presence of the pilot.

\section{NOISE UnCERTAINTY}

\section{A. Motivation}

Noise in most communication systems is an aggregation of various independent sources including not only thermal noise at the receiver, but also interference due to nearby unintended emissions, weak signals from transmitters very far away, etc. By appealing to the Central Limit Theorem (CLT), one usually assumes that the noise at the receiver is a Gaussian random variable. But we know that the error term in the CLT goes to zero only as $\frac{1}{\sqrt{N}}$, where $N$ is the number of independent random variables being summed up to constitute noise (see Thm. 1.4.9 in [1] for details). In real life $N$ is usually moderate and the error term in the CLT should not be neglected. Further, as the CLT is a statement about convergence in distribution, we cannot tell anything about the behavior of the moments of the noise.

The above discussion leads us to the conclusion that the actual noise process is only approximately Gaussian. However, most receivers work under the Gaussian assumption of noise and try to measure the noise variance $\sigma_{n}^{2}$ (Assume that ' $\mathrm{n}$ ' stands for nominal and ' $a$ ' stands for actual in all the subscripts hereafter).

\section{B. Noise uncertainty model}

We know that the receiver tries to estimate the noise variance by taking a large number of samples. But, there will always be some residual uncertainty in estimating the noise variance. We model this residual uncertainty by assuming that the receiver can narrow down the noise process only to within a class of distributions denoted by $\mathcal{W}_{x}$, where $x$ parameterizes the amount of residual uncertainty. We call this set the uncertainty class of noise for a given receiver. To begin with we need to make the following basic assumptions on this set $\mathcal{W}_{x}$ :
- The noise processes in this set must all be 'white', i.e., samples are i.i.d.

- The noise processes must be symmetric, i.e., if $W_{a} \in$ $\mathcal{W}_{x}$ then we assume that $\mathbb{E} W_{a}^{2 k-1}=0$. We make this assumption for physical reasons.

Finally, we need to model the fact that there is at most $x$ $\mathrm{dB}$ of uncertainty in the noise processes in $\mathcal{W}_{x}$. Therefore, for every noise random variable $W_{a} \in \mathcal{W}_{x}$ we assume that

- All the moments of the noise process must be close to the nominal noise moments, i.e., we assume that $\mathbb{E} W_{a}^{2 k} \in\left[\frac{1}{\alpha} \mathbb{E} W_{n}^{2 k}, \alpha \mathbb{E} W_{n}^{2 k}\right]$, where $W_{n} \sim N\left(0, \sigma_{n}^{2}\right)$ is the estimated nominal Gaussian random variable and $\alpha=10^{x / 10}>1$.

Since the uncertain set $\mathcal{W}_{x}$ models post-noise-estimation uncertainty, we require that our detector meets both our target ${ }^{2}$ probability of false alarm $P_{F A}$ and probability of missed detection $P_{M D}$ regardless of which possible noise process actually occurs. Otherwise, we say that detection is impossible with this particular detector under our uncertainty set $\mathcal{W}_{x}$.

For notational convenience, we denote the even moments of a Gaussian random variable by $\mathbb{E} W_{n}^{2 k}=1 \cdot 3 \cdot 5 \cdots(2 k-$ 1) $\sigma_{n}^{2 k} \triangleq(2 k-1) ! ! \sigma_{n}^{2 k}$.

\section{Implications of Noise Uncertainty}

How does noise uncertainty affect detection of signals in low SNR? Before we try to answer this general question, lets review the radiometer case. [14]

Clearly, if $\sigma_{a}^{2}=\sigma_{n}^{2}+P$ the radiometer can be made to believe that the signal is present even when the signal is actually absent. On the flip side, if $\sigma_{a}^{2}+P=\sigma_{n}^{2}$ the radiometer thinks that the signal is absent even when it is actually present. Thus, this example clearly illustrates that if the $S N R$ is sufficiently low, there is enough uncertainty in the noise to render the radiometer useless.

For notational clarity, we use lower case $s n r$ to denote the signal to noise ratio and reserve $S N R$ to denote the signal to noise ratio in decibels. Define $s n r_{\text {wall }}$ to be the maximal $s n r$ such that for every $s n r \leq s n r_{\text {wall }}$ detection is impossible for the particular detector. Now, we try to see if the same threshold behavior as in the case of the radiometer is observed for more sophisticated detectors that look at higher moments of the received signal.

Theorem 1: For the case of detection of a weak unknown BPSK signal sampled at Nyquist and uncertainty set $\mathcal{W}_{x}$, $s n r_{\text {wall }}$ for the $2 k$-th moment detector satisfies

$$
\frac{\alpha-1}{k}\left[1-\frac{\alpha-1}{2-\alpha}\right] \leq s n r_{w a l l}^{(2 k)} \leq \frac{\alpha-1}{k}
$$

where $\alpha=10^{x / 10}$.

Proof: The test statistic for the $2 k$-th moment detector is given by: $T(\vec{Y})=\frac{1}{N} \sum_{i=1}^{N} Y_{i}^{2 k}$, where $Y_{i}$ is the $i$-th received sample. It is easy to see that the detector in consideration fails

\footnotetext{
${ }^{2}$ The target $P_{F A}$ and $P_{M D}$ are parameters that certainly will impact detection performance in terms of sample complexity. However, our primary interest in this paper is in showing detection-impossibility results as a function of SNR. Thus, we will not dwell on the quantitative impact of the chosen $P_{F A}, P_{M D}$ on our results.
} 
if the mean of this test statistic under both the hypothesis are equal. This can happen in the following two ways.

Case I: $\mathbb{E} W_{a}^{2 k}=\mathbb{E}\left[X+W_{n}\right]^{2 k}$, i.e., the actual noise moments are high enough to make the receiver wrongly believe that there is a signal present. We begin by expanding $\mathbb{E}\left[X+W_{n}\right]^{2 k}$ and re-writing the condition as

$$
\mathbb{E} W_{a}^{2 k}=\sum_{i=0}^{k}\left(\begin{array}{c}
2 k \\
2 i
\end{array}\right) \mathbb{E} W_{n}^{2 k-2 i} \mathbb{E} X^{2 i}
$$

Dividing by $\mathbb{E} W_{n}^{2 k}$ on both sides of (3) we get,

$$
\begin{aligned}
\frac{\mathbb{E} W_{a}^{2 k}}{\mathbb{E} W_{n}^{2 k}} & =\sum_{i=0}^{k}\left(\begin{array}{c}
2 k \\
2 i
\end{array}\right)\left(\frac{\mathbb{E} W_{n}^{2 k-2 i}}{\mathbb{E} W_{n}^{2 k}}\right) \mathbb{E} X^{2 i} \\
& =\sum_{i=0}^{k}\left(\begin{array}{c}
2 k \\
2 i
\end{array}\right)\left(\frac{1 \cdot 3 \cdots(2 k-2 i-1)}{1 \cdot 3 \cdots(2 k-1)}\right) \frac{\mathbb{E} X^{2 i}}{\sigma_{n}^{2 i}} \\
& =\sum_{i=0}^{k} \frac{\left(\begin{array}{c}
2 k \\
2 i
\end{array}\right)}{(2 k-2 i+1) \cdots(2 k-1)} s n r^{i} \\
& =1+k \cdot s n r+\cdots+\frac{1}{(2 k-1) ! !} s n r^{k}
\end{aligned}
$$

Until now we have not used the fact that the actual noise random variable $W_{a} \in \mathcal{W}_{x}$, i.e., $\frac{1}{\alpha} \leq \frac{\mathbb{E} W_{a}^{2 k}}{\mathbb{E} W^{2 k}} \leq \alpha$. Since, the right hand side of (4) is greater than 1 , and is also monotonic in $s n r, s n r_{w a l l}^{(2 k)}$ must be a solution to

$$
\begin{aligned}
\alpha & =1+k \cdot s n r+\cdots+\frac{1}{(2 k-1) ! !} s n r^{k} \\
& =\sum_{i=0}^{k} \frac{\left(\begin{array}{c}
2 k \\
2 i
\end{array}\right)}{(2 k-2 i+1) \cdots(2 k-1)} s n r^{i}
\end{aligned}
$$

From (5) it is obvious that $1+k \cdot s n r \leq \alpha$. This simplifies to give $s n r \leq \frac{\alpha-1}{k}$, which is the desired upper bound in (2). To get a lower bound, we substitute this upper bound into (5)

$$
\begin{aligned}
\alpha & \leq \sum_{i=0}^{k} \frac{\left(\begin{array}{l}
2 k \\
2 i
\end{array}\right)}{(2 k-2 i+1) \cdots(2 k-1)}\left(\frac{\alpha-1}{k}\right)^{i} \\
& <1+k \cdot s n r+\sum_{i=2}^{k}(\alpha-1)^{i} \\
& <1+k \cdot s n r+\sum_{i=2}^{\infty}(\alpha-1)^{i} \\
& =1+k \cdot s n r+\frac{(\alpha-1)^{2}}{2-\alpha}
\end{aligned}
$$

In the second inequality above we have used the fact that $\frac{\left(\begin{array}{c}2 k \\ 2 i\end{array}\right)}{(2 k-2 i+1) \cdots(2 k-1)} \frac{1}{k^{i}}<1$ for $k \geq 2$. Re-writing (6) as

$$
k \cdot s n r \geq(\alpha-1)\left[1-\frac{\alpha-1}{2-\alpha}\right]
$$

we get the lower bound in (2).

Case II: $\mathbb{E}\left[W_{n}\right]^{2 k}=\mathbb{E}\left[X+W_{a}\right]^{2 k}$, i.e., the actual noise moments are lower than the receiver's estimate by enough so that the receiver fails to detect the signal. Expanding $\mathbb{E}[X+$ $\left.W_{a}\right]^{2 k}$, we must have

$$
\mathbb{E} W_{n}^{2 k}=\sum_{i=0}^{k}\left(\begin{array}{c}
2 k \\
2 i
\end{array}\right) \mathbb{E} W_{a}^{2 k-2 i} \mathbb{E} X^{2 i}
$$

As in the previous case, dividing by $\mathbb{E} W_{n}^{2 k}$, we get

$$
1=\sum_{i=0}^{k}\left(\begin{array}{c}
2 k \\
2 i
\end{array}\right)\left(\frac{\mathbb{E} W_{a}^{2 k-2 i}}{\mathbb{E} W_{n}^{2 k}}\right) \mathbb{E} X^{2 i}
$$

which simplifies to

$$
\begin{aligned}
1 & =\sum_{i=0}^{k}\left(\begin{array}{c}
2 k \\
2 i
\end{array}\right)\left(\frac{1 \cdot 3 \cdots(2 k-2 i-1)}{1 \cdot 3 \cdots(2 k-1)}\right) \frac{\mathbb{E} X^{2 i}}{\sigma_{n}^{2 i}} \frac{\mathbb{E} W_{a}^{2 k-2 i}}{\mathbb{E} W_{n}^{2 k-2 i}} \\
& =\sum_{i=0}^{k} \frac{\left(\begin{array}{l}
2 k \\
2 i
\end{array}\right)}{(2 k-2 i+1) \cdots(2 k-1)} s n r^{i} \frac{\mathbb{E} W_{a}^{2 k-2 i}}{\mathbb{E} W_{n}^{2 k-2 i}} \\
& =\frac{\mathbb{E} W_{a}^{2 k}}{\mathbb{E} W_{n}^{2 k}}+k \cdot \operatorname{snr} \frac{\mathbb{E} W_{a}^{2 k-2}}{\mathbb{E} W_{n}^{2 k-2}}+\ldots+\frac{1}{(2 k-1) ! !} s n r^{k}
\end{aligned}
$$

Now, using the condition that $W_{a} \in \mathcal{W}_{x}$, we must have $\frac{\mathbb{E} W_{a}^{2 l}}{\mathbb{E} W_{n}^{2 l}} \geq \frac{1}{\alpha}$, for all $l \leq k$. Substituting this in (7) we can easily see that $s n r_{\text {wall }}^{(2 k)}$ must be a solution to

$$
\begin{aligned}
1 & =\frac{1}{\alpha}+k \cdot \operatorname{snr} \frac{1}{\alpha}+\ldots+\frac{1}{(2 k-1) ! !} s n r^{k} \\
\Rightarrow \alpha & =1+k \cdot s n r+\ldots+\frac{1}{(2 k-1) ! !} s n r^{k} \alpha
\end{aligned}
$$

Equation (8) looks very similar to (5). In fact it is exactly identical to it except the ' $\alpha$ ' in the last term on the right hand side of (8). As $\alpha>1$, the snr solution to (8) is strictly smaller than the solution to (5). Therefore, the final $s n r_{\text {wall }}$ must be the solution to equation (5). However, the difference between the solutions to equations (5) and (8) is tiny in the interesting case when $\alpha \approx 1$ and so snr is also low. Thus, the lower bound in (2) continues to hold (See fig. 2).

Remarks: Note that the difference between the derived upper and lower bound (in $\mathrm{dB})$ is $-10 \log _{10}\left[1-\frac{\alpha-1}{2-\alpha}\right] \approx-10(\alpha-$ $1) \log _{10} 2$, for $\alpha \approx 1$. This shows that the gap between our bounds is negligible compared to the value of the $S N R_{\text {wall }}^{(2 k)}$ (Refer to fig. 2). Also, note that the position of $S N R_{\text {wall }}^{(2 k)}$ varies as a logarithm of $k$ and hence checking for large moments buys us very little in terms of detectability .

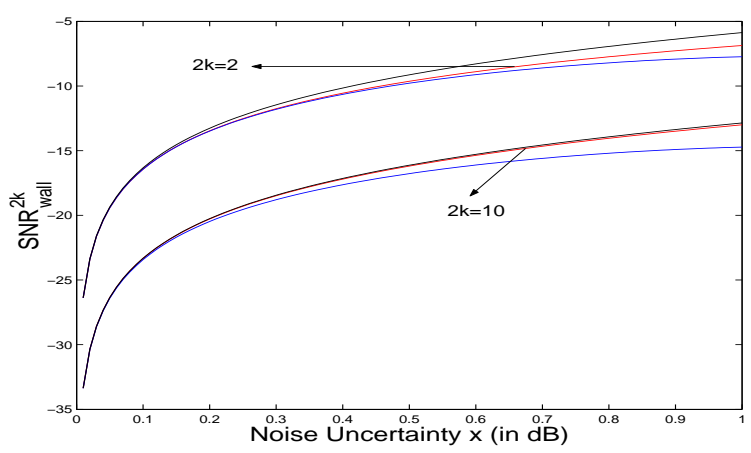

Fig. 2. The location of the $S N R_{\text {wall }}^{(2 k)}$ (in $\mathrm{dB}$ ) for $2 k=2,10$, as a function of the noise uncertainty $x$ (in $\mathrm{dB}$ ) is plotted in this figure. The red curves in the plot have been computed numerically by calculating the root of the equation (5), the blue curves are computed using the lower bound in Theorem 1, and the black curves are plotted using the upper bound in Theorem 1 . 


\section{Discussion of results}

Theorem 1 shows that when the signal to be detected is random, non-detectability is always a problem at low enough SNR's regardless of how many samples we take or which moment we look at. A perfectly known signal, on the other hand, can be detected even under such noise uncertainty since we get coherent processing gain using the matched filter. The signal is isolated, while the noise is averaged. The moments of the averaged noise decrease to zero as the number of samples increases regardless of where it lies in the uncertainty set.

To better illustrate what is different about the random primary signal case, we will consider the $2 k$-th moment detector's test statistic: $T(\vec{Y})=\frac{1}{N} \sum_{i=1}^{N} Y_{i}^{2 k}$ pictorially in Fig. 3a. The horizontal line represents the possible locations of the test statistic $T(\vec{Y})$, and the $2 k$-th moments under both hypotheses are marked as two points on this line. The dotted vertical line represents the threshold $\gamma$ which divides the whole space into two decision regions corresponding to the two hypotheses.

Now, let us see how the picture changes when we consider uncertainty in noise. According to our model for uncertainty, noise can have a set of possible $2 k$-th moments under both hypotheses, which are denoted by intervals in Fig. 3b. For low signal powers, these two intervals must overlap, as shown by the shaded region. Note that whatever threshold $\gamma$ (vertical line in the figure) we choose, the detector cannot guarantee that both $P_{F A}$ and $P_{M D}$ are low enough for all the noise processes in the set $\mathcal{W}_{x}$. Due to the shaded region, at least one of $P_{F A}$ and $P_{M D}$ can be made to go to 1 . Therefore, the moment detectors are useless for detecting signals below the SNR wall.

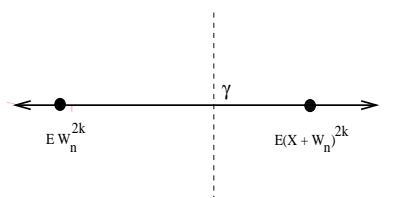

(a)

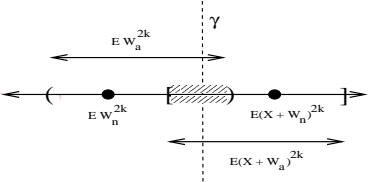

(b)
Fig. 3. This figure pictorially depicts the two hypothesis and the threshold in a $2 k$-th moment detector. The figure on the left shows the case when there is no noise uncertainty and the one on the right is the case when there is noise uncertainty. Note that under noise uncertainty and sufficiently weak signal power, there is an overlap region under both hypotheses, which renders the detector useless.

\section{E. Approaching the SNR wall}

Figure 4 shows the sample complexity of moment detectors near the SNR wall. The green curve shows the sample complexity of the radiometer without noise uncertainty $(N=$ $O\left(1 / S N R^{2}\right)$, slope $\left.=2\right)$. The remaining curves in the figure show the sample complexities of various moment detectors with noise uncertainty. Recall that the test statistic for a $2 k$-th moment detector is given by $T(\vec{Y})=\frac{1}{N} \sum_{i=1}^{N} Y_{i}^{2 k}$, where $Y_{i}$ is the $i$-th received sample. The values of $N$ we are considering here are very large, in fact larger than the number of samples required for the radiometer without noise uncertainty. Also, our target probability of missed detection and false alarm are moderate, i.e., not changing with $N$. Therefore the Central Limit theorem is a good approximation here (Note that the error in the central limit theorem decays as $1 / \sqrt{N}$. [1]).
Hence, we assume that $\frac{T(\vec{Y})-N \mathbb{E} Y_{i}^{2 k}}{\sqrt{N} \operatorname{var}\left(Y_{i}\right)} \sim \mathcal{N}(0,1)$. This reduces the problem into a standard binary hypothesis testing problem with different mean under both hypotheses. Therefore, $N$ is given by:

$N=\left[\frac{Q^{-1}\left(P_{F A}\right) \sqrt{\left(\mathbb{V}\left(Y_{i}^{2 k}\right) \mid H_{0}\right)}-Q^{-1}\left(P_{D}\right) \sqrt{\left(\mathbb{V}\left(Y_{i}^{2 k}\right) \mid H_{1}\right)}}{\mathbb{E}\left(Y_{i}^{2 k} \mid H_{1}\right)-\mathbb{E}\left(Y_{i}^{2 k} \mid H_{0}\right)}\right]^{2}$

where $\mathbb{V}($.$) stands for the variance operator. Recall that the$ detector must hit the target error probabilities uniformly over the whole uncertainty set $\mathcal{W}_{x}$. Therefore, the sample complexity is dominated by the case when the difference in means (denominator term in (9)) in the above equation is minimized.

Thus, the sample complexity required to meet our performance targets uniformly over the uncertain noise tends to infinity as the $S N R$ tends to $S N R_{\text {wall }}^{(2 k)}$. Also note that these performance curves shift to the left by $10 \log k$, which verifies that the upper bound obtained in Thm. 1 is very tight.

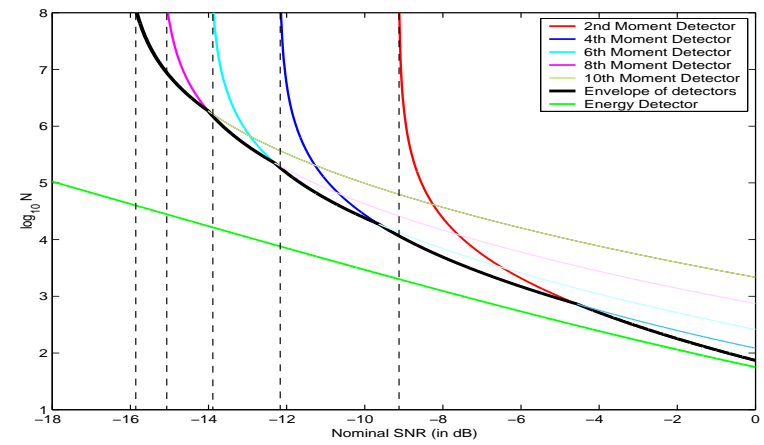

Fig. 4. This figure shows the sample complexity of the moment detectors (number of samples as a function of SNR) at a moderate noise uncertainty of $x=0.5 \mathrm{~dB}$. The curves in this figure have been computed using the equation $(9)$

\section{F. Other possible noise uncertainty models}

One might argue that the results in section III-C arise due to the specific model we used for noise uncertainty, and that they are not fundamental. In this section, we try to show that our model is a minimalist model and any other reasonable noise uncertainty model will lead to an uncertainty class which includes our uncertainty class. Thus in practice, the problem will only get worse.

For example, consider the simple case in which the receiver assumes that the noise is Gaussian, but its estimate of the noise variance is off by some factor, i.e., the receiver estimates the noise variance to be $\sigma_{n}^{2}$, whereas the actual noise variance is $\sigma_{a}^{2}$ $\left(\sigma_{n} \neq \sigma_{a}\right)$. If $\sigma_{n}>\sigma_{a}$ then the ratio of their $2 k$-th moments is $\left(\frac{\sigma_{n}}{\sigma_{a}}\right)^{2 k}$, which goes to infinity as $k \rightarrow \infty$. Conversely, if $\sigma_{n}<\sigma_{a}$ then $\left(\frac{\sigma_{n}}{\sigma_{a}}\right)^{2 k}$ goes to zero as $k \rightarrow \infty$. Therefore we have shown that even such a simple case is not included in our noise model.

Motivated by the above example, we propose the following noise uncertainty model. Suppose that the user estimates the noise to be Gaussian with variance $\sigma_{n}^{2}$, i.e., $W_{n} \sim N\left(0, \sigma_{n}^{2}\right)$. 
Define the new noise uncertainty set $\tilde{\mathcal{W}}_{x}$ to be the set of noise random variables, $W_{a}$ such that the moments of $W_{a}$ are all sandwiched between the corresponding moments of $\frac{1}{\alpha} W_{n}$ and $\alpha W_{n}$, i.e., $\mathbb{E} W_{a}^{2 k} \in\left[\frac{1}{\alpha^{2 k}} \mathbb{E} W_{n}^{2 k}, \alpha^{2 k} \mathbb{E} W_{n}^{2 k}\right]$ where $\alpha=$ $10^{(x / 10)}$. Under this model, we can show that there exists a single threshold below which every moment detector fails to detect the signal [15]. Thus, in this model detection becomes absolutely impossible.

This second uncertainty model is very reasonable in practice, and it clearly contains the model of the previous sections. In the next section, we stay with our original minimalist noise uncertainty model, but add a finite dynamic range limitation to the radio. This will also make detection absolutely impossible at low SNRs.

\section{RECEIVERS WITH FINITE DYNAMIC RANGE}

In practice the front ends of receivers have a finite dynamic range of operation, i.e., the signal coming into the processing unit is bounded in amplitude.

Theorem 2: For detection of an unknown BPSK signal under noise uncertainty and with receivers constrained to operate within a finite dynamic range $(-M, M)$, there exists a single $s n r_{\text {wall }}$ for any possible detector.

Proof: Note that $W_{n} \sim N\left(0, \sigma_{n}^{2}\right)$ and is independent of $X$. Therefore, the random variable $W_{n}+X$ must have a density function. The detector is unable to detect signals iff there exits a pair of noise random variables $W_{a}$ and $W_{n}$ in the set $\mathcal{W}_{x}$ such that $f_{W_{n}}(w)=f_{W_{a}+X}(w)$ or $f_{W_{a}}(w)=f_{W_{n}+X}(w)$, for $w \in(-M, M)$. To prove this result we construct random variables $W_{a} \in \mathcal{W}_{x}$ satisfying the conditions above.

Case I: $f_{W_{a}}(w)=f_{W_{n}+X}(w), \quad$ for $w \in(-M, M)$

We need the noise to have the same density as $f_{W_{n}+X}$ in $(-M, M)$. It is clear that if we don't restrict this equality to within this finite dynamic range, then the ratio of the moments $\frac{\mathbb{E} W_{a}^{2 k}}{\mathbb{E} W^{2 k}} \rightarrow \infty$ as $k \rightarrow \infty$ and hence the constructed distribution will fall outside the uncertainty class. Hence, we equate the densities exactly within the finite dynamic range and try to reduce the moments by adjusting the density outside the finite dynamic range. Also, since the signal is very weak, it is safe to assume that $|X| \ll M$ and hence, $\mathbb{P}\left(\left|W_{a}\right|>M\right)>\mathbb{P}\left(\left|W_{n}\right|>\right.$ $M)$. We complete the construction of $W_{a}$ by defining

$$
f_{W_{a}}(w)=f_{W_{n}}(w)+\frac{\lambda}{2}[\delta(w-M)+\delta(w+M)]
$$

for $w \in(-M, M)^{c}$ where the constant $\lambda \triangleq \mathbb{P}\left(\left|W_{a}\right|>M\right)-$ $\mathbb{P}\left(\left|W_{n}\right|>M\right)$. Fig. 5a illustrates the noise random variable $W_{a}$ 's density. Having constructed this random variable, all we have to check now is whether it actually falls within our uncertainty class, i.e., if $\frac{\mathbb{E} W_{a}^{2 k}}{\mathbb{E} W_{n}^{2 k}} \leq \alpha$.

For this we start off by observing that

$$
\begin{aligned}
\frac{\mathbb{E} W_{a}^{2 k}}{\mathbb{E} W_{n}^{2 k}}= & \frac{1}{\mathbb{E} W_{n}^{2 k}}\left[\mathbb{E} W_{a}^{2 k} \mathrm{I}_{\left|W_{a}\right|<M}+M^{2 k} \mathbb{P}\left(\left|W_{a}\right|=M\right)\right. \\
& \left.+\mathbb{E} W_{a}^{2 k} \mathrm{I}_{\left|W_{a}\right|>M}\right]
\end{aligned}
$$

TABLE I

$k_{m i n}$ AS A FUNCTION OF THE FINITE DYNAMIC RANGE $M$ IS LISTED FOR TWO DIFFERENT VALUES OF THE NOISE UNCERTAINTY $x$. FOR EACH VALUE OF $M, k_{m i n}$ HAS BEEN NUMERICALLY COMPUTED SUCH THAT $\frac{\mathbb{E} W_{a}^{2 k} \mathrm{I}_{\left|W_{a}\right|<M}+M^{2 k} \mathbb{P}\left(\left|W_{a}\right|=M\right)}{\mathbb{E} W_{n}^{2 k}}<(\alpha-1)$. ALSO, THE $S N R_{\text {wall }}$ HAS BEEN COMPUTED FROM EQUATION (15) FOR EACH VALUE OF $k_{m i n}$.

\begin{tabular}{|c|cc|cc|}
\hline $\mathrm{M}$ & $k_{\min }$ & $S N R_{\text {wall }}(\mathrm{x}=0.5 d B)$ & $k_{\min }$ & $S N R_{\text {wall }}(\mathrm{x}=1 d B)$ \\
\hline $3 \sigma_{n}$ & 8 & -18.1 & 6 & -13.6 \\
\hline $4 \sigma_{n}$ & 12 & -19.9 & 10 & -15.8 \\
\hline $5 \sigma_{n}$ & 17 & -21.4 & 15 & -17.6 \\
\hline $6 \sigma_{n}$ & 25 & -23.1 & 22 & -19.2 \\
\hline
\end{tabular}

Bound the first two terms in (11) by

$\frac{M^{2 k}\left[\mathbb{P}\left(\left|W_{a}\right|<M\right)+\mathbb{P}\left(\left|W_{a}\right|=M\right)\right]}{\mathbb{E} W_{n}^{2 k}} \leq \frac{M^{2 k}}{\mathbb{E} W_{n}^{2 k}}=\frac{M^{2 k}}{(2 k-1) ! ! \sigma_{n}^{2 k}} \rightarrow 0$ as $k$ tends to infinity. Since the sequence above tends to zero, there exists some $k_{\min }$ such that $\frac{\mathbb{E} W_{a}^{2 k} I_{\left|W_{a}\right|<M}+M^{2 k} \mathbb{P}\left(\left|W_{a}\right|=M\right)}{\mathbb{E} W_{n}^{2 k}}<(\alpha-1)$ for all $k \geq k_{\text {min }}$ Table I lists a few typical values for $k_{\min }$ as a function of $M$. Now, we can write the third term in (11) as

$$
\frac{\mathbb{E} W_{a}^{2 k} I_{\left|W_{a}\right|>M}}{\mathbb{E} W_{n}^{2 k}}=\frac{\mathbb{E} W_{n}^{2 k} I_{\left|W_{n}\right|>M}}{\mathbb{E} W_{n}^{2 k}} \leq 1
$$

where the first step is true because $W_{a}=W_{n}$ outside the finite dynamic range. Therefore, for $k \geq k_{\min }$ we have

$$
\frac{\mathbb{E} W_{a}^{2 k}}{\mathbb{E} W_{n}^{2 k}} \leq(\alpha-1)+1=\alpha
$$

where the first term in the inequality follows from the definition of $k_{\min }$ and the second term follows from (12). Now, for $k<k_{\min }$, we use the fact that,

$$
\frac{\mathbb{E} W_{a}^{2 k}}{\mathbb{E} W_{n}^{2 k}} \leq \frac{\mathbb{E}\left(X+W_{n}\right)^{2 k}}{\mathbb{E} W_{n}^{2 k}}
$$

Note, that the above inequality is true for all $k$, but it gives meaningful results only for $k \leq k_{\text {min }}$. From Thm. 1, it is clear that $\frac{\mathbb{E}\left(X+W_{n}\right)^{2 k}}{\mathbb{E} W_{n}^{2 k}} \leq \alpha$ iff $s n r \leq s n r_{\text {wall }}^{2 k}$. Since we want this to hold true for all $k \leq k_{\min }$, we must have $s n r \leq s n r_{w a l l}^{2 k_{\min }}$. Using the lower bound in (2), we get

$$
\text { snr } \leq \frac{\alpha-1}{k_{\min }}\left[1-\frac{\alpha-1}{2-\alpha}\right]
$$

Now, from (13) and (15) it is clear that if snr < $\frac{\alpha-1}{k_{m i n}}\left[1-\frac{\alpha-1}{2-\alpha}\right]$, then $\frac{\mathbb{E} W_{a}^{2 k}}{\mathbb{E} W_{n}^{2 k}} \leq \alpha$ for all $k$, which implies that $W_{a} \in \mathcal{W}_{x}$. Therefore, we have shown that there exists an snr threshold below which detection is absolutely impossible. Thus, we encounter absolute walls due to noise uncertainty under finite dynamic range operation.

$$
\text { Case II: } f_{W_{n}}(w)=f_{W_{a}+X}(w), \quad w \in(-M, M)
$$

In this case the receiver is fooled to believe that the signal is absent even when the signal is actually present. In this case, we construct a noise random variable $W_{a} \in \mathcal{W}_{x}$ satisfying the condition (16). Begin by choosing $W_{a}$ such that $f_{W_{n}}=f_{W_{a}+X}$ within $(-M, M)$. In this case the mass in 


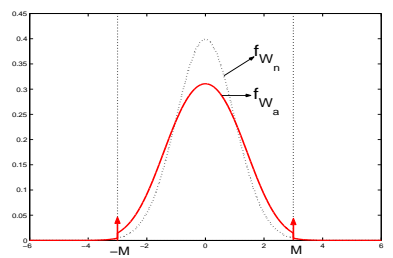

(a)

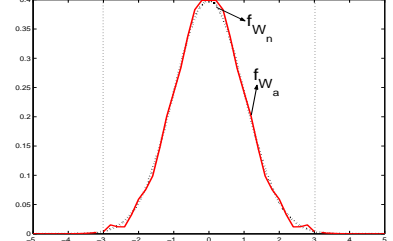

(b)
Fig. 5. The density functions of the constructed noise random variable in both cases to prove Thm. 2 is shown in this figure. The figure on the left corresponds to Case I discussed in section IV. Note that there are two delta functions in the density of $W_{a}$ at $\pm M$. The figure on the right corresponds to case II in section IV. In this figure note that there is a small hole in the density function of $W_{a}$ at $\pm M$. In both these figures, the red curve corresponds to the density function of $W_{a}$ and the black curve corresponds to the density function of $W_{n}$.

$W_{a}$ is smaller than the mass in $W_{n}$ outside the finite dynamic range $(-M, M)$. Therefore, the delta function approach of case I will not work. But, this problem can be overcome by making $f_{W_{a}}(w)=f_{W_{n}}(w)$ for $w \in\left(-M_{1}, M_{1}\right)^{c}$, where we choose $M_{1}$ such that $\mathbb{P}\left(\left|W_{a}\right|>M_{1}\right)=\mathbb{P}\left(\left|W_{n}\right|>M_{1}\right)$. Now, we need to verify that $W_{a} \in \mathcal{W}_{x}$. This can be done exactly as in the proof for case I. Therefore as in case I, if $s n r<\frac{\alpha-1}{k_{\min }}\left[1-\frac{\alpha-1}{2-\alpha}\right]$, detection is absolutely impossible.

Remarks: Theorem 2 shows that the seeming gain coming from using higher moment detectors is illusory. This is because higher moment detectors use rare large values of the test statistic for detection, but a finite dynamic range of operation prevents this.

\section{CONCLUSION AND COMMENTS}

In this paper we have considered fundamental bounds to the detection of signals in very low SNR while subject to uncertainty about the noise. While we have assumed that the signal and the noise are independent of each other and that the noise is white, we assume that we know the distribution of the noise only to within some uncertain set. Even with a minimalist model of the noise uncertainty, we found that this implies a fundamental bound on the detectable SNR if we assume that the radio can only observe the wireless signals and noise to some finite dynamic range.

While our arguments have been focussed on the BPSK transmission case, both Thm. 1 and 2 are not limited to this case. It is not hard to extend our proofs to general zero-mean signal constellations with low signal amplitude. In addition, we believe that these results reflect the generic difficulty of the low SNR signal detection problem and not peculiarities of our model. For example, let us consider the case when the signal is no longer white. ${ }^{3}$ In that case, it is possible to use feature detectors (see [5], [6]) to exploit the colored nature of the signal. However, in such cases, it will also be natural to consider our noise as being only approximately white. After all, it reflects the sum of many different physical sources of undesired signals, not all of which are white! For low enough

\footnotetext{
${ }^{3}$ This just means that we are not going to sample it faster than the Nyquist
}

SNR, we suspect the structure brought by the signal will be indistinguishable from the uncertain low level structure of the noise.

These sorts of fundamental bounds make us further appreciate the usefulness and robustness of the coherent signal processing possible whenever the primary signal has known training data or pilot tones. Coherent processing enables us to take long averages that bring the SNR up to a reasonable value for detection.

\section{REFERENCES}

[1] Probability: Theory and Examples, 3rd ed. Belmont: Duxbury Press, 2004.

[2] R. W. Broderson, A. Wolisz, D. Cabric, S. M. Mishra, and D. Willkomm, "(2004) white paper: Corvus: A cognitive radio approach for usage of virtual unlicensed spectrum." [Online]. Available: http: //bwrc.eecs.berkeley.edu/Research/MCMA/CR_White_paper_final1.pdf

[3] FCC, "Et docket no. 03-237," November 2003. [Online]. Available: http://hraunfoss.fcc.gov/edocs_public/attachmatch/FCC-03-289A1.pdf

[4] — "Et docket no. 03-322," December 2003. [Online]. Available: http://hraunfoss.fcc.gov/edocspublic/attachmatch/FCC-03-322A1.pdf

[5] W. A. Gardner, "Signal interception: Performance advantages of cyclicfeature detectors," IEEE Trans. Commun., vol. 40, pp. 149-159, January 1992.

[6] _ - "Signal interception: A unifying theoretical framework for feature detection," IEEE Trans. Commun., vol. 36, pp. 897-906, August 1998.

[7] N. Hoven and A. Sahai, "Power scaling for cognitive radio," in WirelessCom Symposium on Emerging Networks, Technologies and Standards, June 2005.

[8] T. Kailath and V. Poor, "Detection of stochastic processes," IEEE Trans. Inform. Theory, vol. 44, pp. 2230-2259, October 1998.

[9] D. Middleton, "On the detection of stochastic signals in additive normal noise - part i," IEEE Trans. Inform. Theory, vol. 3, pp. 86-121, June 1957.

[10] I. J. Mitola, "Software radios: Survey, critical evaluation and future directions," IEEE Aerosp. Electron. Syst. Mag, vol. 8, pp. 25-36, April 1993.

[11] R. Price and N. Abramson, "Detection theory," IEEE Trans. Inform. Theory, vol. 7, pp. 135-139, July 1961.

[12] A. Sahai, N. Hoven, and R. Tandra, "Some fundamental limits on cognitive radio," in Forty-Second Allerton Conference on Communication, Contol and Computing, September 2004.

[13] D. Slepian, "Some comments on the detection of gaussian signals in gaussian noise," IEEE Trans. Inform. Theory, vol. 4, pp. 65-68, June 1958.

[14] A. Sonnenschein and P. M. Fishman, "Radiometric detection of spreadspectrum signals in noise of uncertain power," IEEE Trans. Aerosp. Electron. Syst., vol. 28, pp. 654-660, July 1992.

[15] R. Tandra, "Fundamental limits on detection in low snr," Master's thesis, University of California, Berkeley, 2005.

[16] H. Urkowitz, "Energy detection of unknown deterministic signals," Proc. IEEE, vol. 55, pp. 523-531, 1967. 